TRANSACTIONS OF THE

AMERICAN MATHEMATICAL SOCIETY

Volume 361, Number 9, September 2009, Pages 4581-4591

S 0002-9947(09)04640-6

Article electronically published on April 14, 2009

\title{
REGULARITY OF SUBELLIPTIC MONGE-AMPÈRE EQUATIONS IN THE PLANE
}

\author{
PENGFEI GUAN AND ERIC SAWYER
}

\begin{abstract}
We establish a $C^{\infty}$ regularity result for $C^{1,1}$ solutions of degenerate Monge-Ampère equation in $\mathbb{R}^{2}$, under the assumption that the trace of the Hessian is bounded from below.
\end{abstract}

\section{INTRODUCTION}

There is a vast body of elliptic regularity results for the Monge-Ampère equation

$$
\begin{aligned}
\operatorname{det} D^{2} u(x) & =k(x), & & x \in \Omega, \\
u(\xi) & =\varphi(\xi), & & \xi \in \partial \Omega .
\end{aligned}
$$

If the data $k, \varphi, \partial \Omega$ are smooth and $\partial \Omega$ is strictly convex, then there is a unique solution $u$ of (1.1), smooth up to the boundary of $\Omega$ (e.g., 2]).

In the event that ellipticity degenerates, i.e. $k \geq 0$ vanishes in $\Omega$, existence and uniqueness persist with some restrictions, but the optimal regularity of solutions is now $C^{1,1}$ (e.g., see [5, 7]). For the case $n=2$, the Monge-Ampère equation is closely related to Weyl's isometric embedding of surfaces ([10]). If the Gauss curvature is only assumed to be nonnegative, a $C^{1,1}$ isometric embedding was obtained in [6, 8, It is desirable to know under what conditions the solution is smooth. From an analytic point of view, detecting smoothness from the data is problematic even for the simplest degeneracies: the nonsmooth function $u(x)=c_{n}|x|^{2+\frac{2}{n}}$ solves (1.1) with polynomial data $k(x)=|x|^{2}, \varphi(\xi)=c_{n}$ and $\partial \Omega=\mathbb{S}^{n-1}$. A similar global example for the Weyl isometric embedding problem can be found in [1, 9, where the metric is analytic with nonnegative Gauss curvature but the isometric embedding is not $C^{3}$. The problem is that the mean curvature of the isometric embedding vanishes along with the Gauss curvature at a point. That is, the surface is umbilical at a point where the Gauss curvature vanishes.

An alternative approach to detecting smoothness in the degenerate case has been introduced by one of the authors that involves geometric quantities associated with the solution $u$. For example ([4), if in $n=2$ dimensions a $C^{1,1}$ convex solution $u$ to

$$
\operatorname{det} D^{2} u(x)=k(x), \quad x \in \Omega,
$$

Received by the editors April 26, 2007.

2000 Mathematics Subject Classification. Primary 35J60, 35B65.

Research of the authors was supported in part by NSERC Discovery Grants.

(C)2009 American Mathematical Society 
has one fixed principle entry of the Hessian uniformly bounded below by a positive constant, then $u$ is smooth if $k$ is smooth and of appropriately finite type. More precisely, $k(x) \approx x_{1}^{2 m}+B x_{2}^{2 \ell}, \ell \geq m, B \geq 0$ and $u_{22} \geq c>0$ a.e. in $\Omega$.

One of the original motivations in [4] was to study higher regularity of the isometric embedding obtained in [6, 8. However, it was not fulfilled in [4 at that time. A natural conjecture left unresolved in 4 is that the uniform entry of the Hessian assumption can be relaxed to simply bounding the trace of the Hessian from below almost everywhere. Or in the isometric embedding case, the assumption can be relaxed to a lower positive bound on the mean curvature. The purpose of this short note is to establish this conjecture, thus completing the regularity program in the plane begun in [4, as well as answering Question 2 in [6]. Weaker results in higher dimensions appear in [11] and [12].

Theorem 1. Suppose $\Omega \subset \mathbb{R}^{2}$ and that $u(x)$ is a convex $C^{1,1}(\Omega)$ solution to

$$
\operatorname{det} D^{2} u(x)=k(x, u(x), D u(x)), \quad x \in \Omega,
$$

where $k$ is smooth and there is $m \in \mathbb{N}$ and $C>0$ such that

$$
k(x, r, \mathbf{p}) \approx|x|^{2 m} \text { for }(x, r, \mathbf{p}) \text { in compact subsets of } \Omega \times \mathbb{R} \times \mathbb{R}^{n} .
$$

Then $u$ is smooth in $\Omega$ if $\Delta u$ is bounded below by a positive constant $c$ in $\Omega$, i.e.

$$
\triangle u(x) \geq c>0 \text { for a.e. } x \in \Omega .
$$

We also have the following regularity result for the isometric embedding problem.

Theorem 2. Suppose $(M, g)$ is a smooth compact oriented surface with Gauss curvature $\kappa \geq 0$. If $\kappa$ satisfies the finite type condition (1.4) near every vanishing point and if the $C^{1,1}$ isometric embedding (obtained in [6, 8]) $X: M \rightarrow \mathbb{R}^{3}$ has mean curvature $H$ bounded below by a positive constant c almost everywhere, then the embedding $X$ is in fact smooth everywhere.

Theorem 1 follows immediately from the result in [4] mentioned above (see Theorem 3 and Remark 21 (ii) there), together with Lemma 1 in the next section which proves the continuity of the eigenvector $v(x)$ of $D^{2} u(x)$ with least eigenvalue, and then deduces that in an appropriate coordinate system $u_{22} \geq \frac{1}{2}$ in a neighbourhood of the origin. Note also that when $m=1$, the elementary inequality $4 \operatorname{det} D^{2} u \leq(\Delta u)^{2}$ implies $\triangle u \geq C|x|$. If $u$ is $C^{3, \alpha}, \Delta u$ is a nonnegative $C^{1, \alpha}$ function and we must have $\Delta u \geq c$. This shows that (1.5) is a necessary condition for smoothness of $u$. Theorem 2 will also follow from Lemma 1, but we will need to work a bit harder to prove the lemma in this geometric situation.

Added in proof. In a recent preprint [3, Daskalopoulos and Savin proved some sharp $C^{2, \alpha}$ regularity results for equation (1.3) with $k=|x|^{\alpha} g(x)$ for Hölder continuous $g>0$ and without condition (1.5).

\section{Proofs}

For $x$ sufficiently close to the origin, under assumption (1.4), the eigenvalues $\lambda_{1}(x) \leq \lambda_{2}(x)$ of $D^{2} u(x)$ are distinct, with $\lambda_{1}(x) \approx k(x) \approx|x|^{2 m}$ and $\lambda_{2}(x) \approx$ 1. The unit eigenvector $v(x)$ corresponding to the least eigenvalue $\lambda_{1}(x)$ is thus uniquely determined modulo multiplication by \pm 1 , i.e. as a point in the projective space $\mathbb{P}^{1}$. The following lemma is crucial. 
Lemma 1. Suppose $\Omega \subset \mathbb{R}^{2}$, that $u(x)$ is a convex $C^{1,1}(\Omega)$ solution to (1.3) with $k$ as in (1.4), and that $\triangle u(x) \geq 1$. If for $x \in \Omega \backslash\{0\}$ sufficiently close to the origin, $v(x)$ denotes the eigenvector of $D^{2} u(x)$ with least eigenvalue, then $v(x)$ admits a continuous extension in $\mathbb{P}^{1}$ to 0 , and in coordinates with $v(0)= \pm(1,0)$ we have that $u_{22}(x) \geq \frac{1}{2}>0$ in a neighbourhood of the origin.

Proof of Lemma 1. It's well known that $u$ is smooth in $\Omega \backslash\{0\}$. For $|x|$ sufficiently small the least eigenvalue $\lambda(x)$ of $D^{2} u(x)$ satisfies $\lambda(x) \approx|x|^{2 m}$. If we view the corresponding eigenvector $v(x)$ as a point in $\mathbb{P}^{1}$, then $v$ is smooth in $\Omega \backslash\{0\}$.

Step 1. For a convex $C^{1,1}(\Omega)$ solution $u$ to (1.3) with $k$ smooth as in (1.4), let

$$
\sigma=\sum_{a, b, c, d, e, f=1}^{2} u^{a d} u^{b e} u^{c f} u_{a b c} u_{d e f}
$$

be the square of the third order derivatives of $u$ with respect to the metric $\left[u^{i j}\right]=$ $\left[u_{i j}\right]^{-1}$. Proposition 2 in [12] uses Calabi's identity to obtain the estimate

$$
k(x) \sigma(x) \leq C|x|^{-2},
$$

where $C$ depends on $\|u\|_{C^{1,1}}$. See also the lemma in section 4 of Iaia 9 for the inequality $k^{2} \sigma \leq C$, which coincides with (2.1) when $m=1$, but is weaker otherwise. In coordinates that diagonalize $D^{2} u$ at $x_{0} \neq 0$, we may pick a representative of $v$ such that $v\left(x_{0}\right)=(1,0)$. Then (2.1) yields $\sum \frac{u_{a b c}^{2}}{u_{a a} u_{b b} u_{c c}}=\sigma\left(x_{0}\right) \leq C k\left(x_{0}\right)^{-1}\left|x_{0}\right|^{-2}$ and hence

$$
\begin{aligned}
D^{2} u\left(x_{0}\right) & =\left[\begin{array}{cc}
u_{11}\left(x_{0}\right) & 0 \\
0 & u_{22}\left(x_{0}\right)
\end{array}\right] \\
\left|u_{11}\left(x_{0}\right)\right| & \approx k\left(x_{0}\right) \approx\left|x_{0}\right|^{2 m} \\
\left|u_{22}\left(x_{0}\right)\right| & \approx 1 \\
\left|u_{111}\left(x_{0}\right)\right| & \leq C k\left(x_{0}\right)^{-\frac{1}{2}}\left|x_{0}\right|^{-1} u_{11}\left(x_{0}\right)^{\frac{3}{2}} \leq C\left|x_{0}\right|^{2 m-1}, \\
\left|u_{112}\left(x_{0}\right)\right| & \leq C k\left(x_{0}\right)^{-\frac{1}{2}}\left|x_{0}\right|^{-1} u_{11}\left(x_{0}\right) \leq C\left|x_{0}\right|^{m-1}, \\
\left|u_{122}\left(x_{0}\right)\right| & \leq C k\left(x_{0}\right)^{-\frac{1}{2}}\left|x_{0}\right|^{-1} u_{11}\left(x_{0}\right)^{\frac{1}{2}} \leq C\left|x_{0}\right|^{-1}, \\
\left|u_{222}\left(x_{0}\right)\right| & \leq C k\left(x_{0}\right)^{-\frac{1}{2}}\left|x_{0}\right|^{-1} \leq C\left|x_{0}\right|^{-m-1} .
\end{aligned}
$$

If we keep these coordinates fixed, we get for a nearby point $x$,

$$
\begin{aligned}
D^{2} u(x) & =\left[\begin{array}{ll}
u_{11}(x) & u_{12}(x) \\
u_{12}(x) & u_{22}(x)
\end{array}\right], \\
v(x) & =\left[\begin{array}{cc}
\cos \theta & -\sin \theta \\
\sin \theta & \cos \theta
\end{array}\right] v\left(x_{0}\right),
\end{aligned}
$$

where $\theta$ is determined by

$$
\left[\begin{array}{cc}
\cos \theta & \sin \theta \\
-\sin \theta & \cos \theta
\end{array}\right]\left[\begin{array}{ll}
u_{11}(x) & u_{12}(x) \\
u_{12}(x) & u_{22}(x)
\end{array}\right]\left[\begin{array}{cc}
\cos \theta & -\sin \theta \\
\sin \theta & \cos \theta
\end{array}\right]=\left[\begin{array}{ll}
a & 0 \\
0 & d
\end{array}\right]
$$


$a \approx|x|^{2 m}$ and $d \approx 1$. Indeed, if $R_{\theta}$ denotes rotation by angle $\theta$, then

$$
\begin{aligned}
D^{2} u(x) v(x) & =R_{\theta}\left\{R_{-\theta} D^{2} u(x) R_{\theta}\right\} v\left(x_{0}\right) \\
& =\left[\begin{array}{cc}
\cos \theta & -\sin \theta \\
\sin \theta & \cos \theta
\end{array}\right]\left[\begin{array}{ll}
a & 0 \\
0 & d
\end{array}\right]\left(\begin{array}{l}
1 \\
0
\end{array}\right) \\
& =a\left[\begin{array}{cc}
\cos \theta & -\sin \theta \\
\sin \theta & \cos \theta
\end{array}\right]\left(\begin{array}{l}
1 \\
0
\end{array}\right)=a v(x) .
\end{aligned}
$$

Solving (2.3) for $\theta$ we get

$$
\tan 2 \theta=-\frac{2 u_{12}(x)}{u_{22}(x)-u_{11}(x)} .
$$

Thus we see that for $x$ sufficiently close to $x_{0}, \theta$ is small, $\left|u_{22}(x)-u_{11}(x)\right| \geq \frac{1}{2}$ and

$$
\left|v(x)-v\left(x_{0}\right)\right|=2\left|\sin \frac{\theta}{2}\right| \approx|\theta| \approx\left|u_{12}(x)\right|=\left|u_{12}(x)-u_{12}\left(x_{0}\right)\right|,
$$

in coordinates that diagonalize $D^{2} u$ at $x_{0}$.

Step 2. Now consider the vector fields

$$
X(x)=D_{v(x)} \text { and } Y(x)=\sqrt{k(x)} D_{v(x)^{\perp}},
$$

where $v(x)^{\perp}$ is $v(x)$ rotated through $90^{\circ}$. From (1.4) and (2.2) we get

$$
\begin{aligned}
\left|X u_{12}\left(x_{0}\right)\right| & =\left|u_{112}\left(x_{0}\right)\right| \leq C\left|x_{0}\right|^{m-1}, \\
\left|Y u_{12}\left(x_{0}\right)\right| & =\sqrt{k\left(x_{0}\right)}\left|u_{212}\left(x_{0}\right)\right| \leq C\left|x_{0}\right|^{m-1}
\end{aligned}
$$

still in coordinates that diagonalize $D^{2} u$ at $x_{0}$. We now claim that

$$
\begin{aligned}
&\left|X v\left(x_{0}\right)\right| \leq\left|u_{112}\left(x_{0}\right)\right| \leq C\left|x_{0}\right|^{m-1}, \\
&\left|Y v\left(x_{0}\right)\right| \leq \sqrt{k\left(x_{0}\right)}\left|u_{122}\left(x_{0}\right)\right| \leq C\left|x_{0}\right|^{m-1},
\end{aligned}
$$

in coordinates that diagonalize $D^{2} u$ at $x_{0}$. Indeed, with $v_{0}=v\left(x_{0}\right)$ and $v_{0}^{\perp}=$ $v\left(x_{0}\right)^{\perp}$, inequality (2.5) yields

$$
\begin{aligned}
\left|\frac{v\left(x_{0}+h v_{0}\right)-v\left(x_{0}\right)}{h}\right| & \leq C\left|\frac{u_{12}\left(x_{0}+h v_{0}\right)-u_{12}\left(x_{0}\right)}{h}\right|, \\
\sqrt{k\left(x_{0}\right)}\left|\frac{v\left(x_{0}+h v_{0}^{\perp}\right)-v\left(x_{0}\right)}{h}\right| & \leq C \sqrt{k\left(x_{0}\right)}\left|\frac{u_{12}\left(x_{0}+h v_{0}^{\perp}\right)-u_{12}\left(x_{0}\right)}{h}\right|,
\end{aligned}
$$

and letting $h \rightarrow 0$ (recall that $v$ and $u_{12}$ are smooth away from the origin) we get $\left|X v\left(x_{0}\right)\right|=\left|D_{v_{0}} v\left(x_{0}\right)\right| \leq C\left|D_{v_{0}} u_{12}\left(x_{0}\right)\right|=C\left|u_{112}\left(x_{0}\right)\right|$, $\left|Y v\left(x_{0}\right)\right|=\sqrt{k\left(x_{0}\right)}\left|D_{v_{0}^{\perp}} v\left(x_{0}\right)\right| \leq C \sqrt{k\left(x_{0}\right)}\left|D_{v_{0}^{\perp}} u_{12}\left(x_{0}\right)\right|=C \sqrt{k\left(x_{0}\right)}\left|u_{122}\left(x_{0}\right)\right|$.

Step 3. Next we claim that

$$
\int_{B(0, R)}|\nabla v(x)|^{2} d x<\infty
$$


for all balls $B(0, R) \subset \Omega$ (note that the Calabi estimate (2.7) yields only $|\nabla v(x)| \leq$ $\left.C|x|^{-1}\right)$. For this we first establish the inequality

$$
\int_{B(0, R)}\left\{\sum_{i, j=1}^{2} c^{i j}(\triangle u)_{i}(\triangle u)_{j}\right\} \leq C,
$$

where $c^{i j}=\left(\operatorname{det} D^{2} u\right) u^{i j}=k G u^{i j}$. We start with the identity

$$
\mathcal{L}(\triangle u) \equiv \sum_{i, j=1}^{2} u^{i j}(\triangle u)_{i j}=\sum_{i, j, a, b, \ell=1}^{2} u^{i a} u^{b j} u_{a b \ell} u_{i j \ell}+\triangle \log (k G),
$$

and multiply through by $k G \triangle u$ to obtain

$$
\begin{aligned}
(\triangle u) \sum_{i, j=1}^{2} c^{i j}(\triangle u)_{i j} & =(\triangle u) \sum_{i, j, a, b, \ell=1}^{2} k G u^{i a} u^{b j} u_{a b \ell} u_{i j \ell}+(\triangle u) k G \triangle \log (k G) \\
& \geq k G\{\triangle \log (k)+\triangle \log (G)\} \\
& \geq-C+k G \frac{1}{G}\{\triangle D u-C\} \geq-C
\end{aligned}
$$

for some $C>0$, since $(2.2)$ implies $k(x)|\triangle D u(x)| \leq C|x|^{2 m}|x|^{-m-1} \leq C$. The rows and columns of $\left[c^{i j}\right]_{i j=1}^{2}$ are divergence free, and so integration by parts yields

$$
-\sum_{i, j=1}^{2} \int_{\Gamma}(\triangle u)_{j} c^{i j}(\triangle u)_{i}+\sum_{i, j=1}^{2}\left\{\int_{\partial B(0, R)}+\int_{\partial B(0, \varepsilon)}\right\} \mathbf{n}_{j}(\triangle u) c^{i j}(\triangle u)_{i} \geq-C,
$$

for $0<\varepsilon<R$ and $\Gamma=B(0, R) \backslash B(0, \varepsilon)$, where $\mathbf{n}$ denotes the unit outward normal to $\partial \Gamma$ and $\mathbf{n}_{j}$ is the component of $\mathbf{n}$ in the $j^{\text {th }}$ direction.

Now on $\partial B(0, r)$, if we fix a point $z$ with $|z|=r$ and choose coordinates that diagonalize $D^{2} u(z)$ with $u_{11}(z) \approx k(z)$, then we have $\left[\begin{array}{cc}c^{11} & c^{12} \\ c^{21} & c^{22}\end{array}\right]=\left[\begin{array}{cc}u_{22} & 0 \\ 0 & u_{11}\end{array}\right]$, and so at $z$ (2.2) yields

$$
\begin{aligned}
\left|\sum_{i, j=1}^{2} \mathbf{n}_{j}(\triangle u) c^{i j}(\triangle u)_{i}\right| & \leq C\left\{u_{22}\left|u_{111}+u_{221}\right|+u_{11}\left|u_{112}+u_{222}\right|\right\} \\
& \leq C\left(r^{2 m-1}+r^{-1}\right)+C r^{2 m}\left(r^{m-1}+r^{-m-1}\right) \leq C r^{-1} .
\end{aligned}
$$

Thus we have

$$
\left|\sum_{i, j=1}^{2} \int_{\partial B(0, r)} \mathbf{n}_{j}(\triangle u) c^{i j}(\triangle u)_{i}\right| \leq C \frac{1}{r}|\partial B(0, r)| \leq C .
$$

Altogether then, with $r$ equal to both $R$ and $\varepsilon$, we obtain (2.9) if we let $\varepsilon \rightarrow 0$.

To obtain (2.8) from this we recall from (2.7) that

$$
\left|D_{v(x)^{\perp}} v(x)\right| \leq C\left|u_{122}(x)\right|
$$


in coordinates that diagonalize $D^{2} u$ at $x$. In the same coordinates we also have

$$
\begin{aligned}
\sum_{i, j=1}^{2} c^{i j}(\triangle u)_{i}(x)(\triangle u)_{j}(x) & =u_{22}\left(u_{111}+u_{221}\right)^{2}+u_{11}\left(u_{112}+u_{222}\right)^{2} \\
& \geq u_{22}(x)\left(u_{111}(x)+u_{221}(x)\right)^{2} \\
& \geq c u_{221}(x)^{2}-C
\end{aligned}
$$

from (2.2). Altogether we have

$$
\left|D_{v(x)^{\perp}} v(x)\right|^{2} \leq C \sum_{i, j=1}^{2} c^{i j}(\triangle u)_{i}(x)(\triangle u)_{j}(x)+C,
$$

where the right hand side is independent of rotation of coordinates, and so

$$
\int_{B(0, R)}\left|D_{v(x)^{\perp}} v(x)\right|^{2} d x \leq C+\sum_{i, j=1}^{2} \int_{B(0, R)} c^{i j}(\triangle u)_{i}(\triangle u)_{j} \leq C,
$$

by (2.9). We also have from (2.7) that

$$
\left|D_{v(x)} v(x)\right|=|X v(x)| \leq C|x|^{m-1} .
$$

Thus both $D_{v(x)} v(x)$ and $D_{v(x)^{\perp}} v(x)$ are in $L^{2}(B(0, R))$, and this completes our proof of (2.8).

Step 4. Now we prove the following modulus of continuity for $v$ at the origin: there is $C>0$ such that

$$
\sup _{x_{0}, y_{0} \in B(0, r) \backslash\{0\}}\left|v\left(x_{0}\right)-v\left(y_{0}\right)\right| \leq C r^{m}+C\left\{\int_{B(0, C r)}|\nabla v(x)|^{2}\right\}^{\frac{1}{2}}, \quad 0<r<R .
$$

First let $\gamma(t)$ be an integral curve with respect to the vector field $X$, i.e. $\gamma^{\prime}(t)=$ $v(\gamma(t))$. We have from (2.7) that

$$
\left|\gamma^{\prime \prime}(t)\right|=\left|\frac{d}{d t} v(\gamma(t))\right|=|X v(\gamma(t))| \leq C|\gamma(t)|^{m-1}
$$

gives an upper bound on the curvature of $\gamma(t)$ since $\left|\gamma^{\prime}(t)\right|=|v(\gamma(t))|=1$. Now fix $r$ and $x_{0}, y_{0} \in B(0, r) \backslash\{0\}$ with $\left|x_{0}-y_{0}\right|<\delta r$, where $0<\delta<1$ is fixed sufficiently small, and let $\gamma_{x_{0}}$ (respectively $\gamma_{y_{0}}$ ) be the integral curve of $X$ with $\gamma_{x_{0}}(0)=x_{0}\left(\right.$ respectively $\left.\gamma_{y_{0}}(0)=y_{0}\right)$.

Since $X$ is a unit vector field and the integral curves have bounded curvature (by (2.11)), we can pick parameter intervals $(a, b)$ and $(c, d)$ so that in a properly rotated coordinate system, the images $\gamma_{x_{0}}(a, b)$ and $\gamma_{y_{0}}(c, d)$ have the following properties: there are functions $f$ and $g$ on $(\alpha, \beta)$ with $\beta-\alpha \approx r$ such that $\gamma_{x}(a, b)$ is the graph of $f$ and $\gamma_{y}(c, d)$ is the graph of $g$, i.e.

$$
\gamma_{x_{0}}(a, b)=\{(s, f(s)): \alpha<s<\beta\} \text { and } \gamma_{y_{0}}(c, d)=\{(s, g(s)): \alpha<s<\beta\},
$$

and where

$$
|f(s)-g(s)| \leq C r, \quad \alpha<s<\beta .
$$


We now write

$$
\begin{aligned}
v\left(x_{0}\right)-v\left(y_{0}\right)= & \frac{1}{\beta-\alpha} \int_{\alpha}^{\beta}\left\{v\left(x_{0}\right)-v(s, f(s))\right\} d s \\
& +\frac{1}{\beta-\alpha} \int_{\alpha}^{\beta}\{v(s, f(s))-v(s, g(s))\} d s \\
& +\frac{1}{\beta-\alpha} \int_{\alpha}^{\beta}\left\{v(s, g(s))-v\left(y_{0}\right)\right\} d s \\
= & I+I I+I I I .
\end{aligned}
$$

Now for each $s$ there is $t$ so that using (2.7) we have

$$
\begin{aligned}
\left|v\left(x_{0}\right)-v(s, f(s))\right| & =\left|v\left(x_{0}\right)-v\left(\gamma_{x}(t)\right)\right| \\
& =\left|\int_{0}^{t} \frac{d}{d \sigma} v\left(\gamma_{x}(\sigma)\right) d \sigma\right|=\left|\int_{0}^{t} X v\left(\gamma_{x}(\sigma)\right) d \sigma\right| \leq C r^{m},
\end{aligned}
$$

and we get

$$
|I|+|I I I| \leq C r^{m}
$$

Using

$$
|v(s, f(s))-v(s, g(s))| \leq\left|\int_{g(s)}^{f(s)}\right| \nabla v(s, \tau)|d \tau|,
$$

we get

$$
\begin{aligned}
|I I| & \leq \frac{1}{\beta-\alpha} \int_{\alpha}^{\beta}\left|\int_{g(s)}^{f(s)}\right| \nabla v(s, \tau)|d \tau| d s \\
& \leq \frac{1}{\beta-\alpha} \int_{B(0, C r)}|\nabla v(x)| d x \\
& \leq \frac{1}{\beta-\alpha}|B(0, C r)|^{\frac{1}{2}}\left\{\int_{B(0, C r)}|\nabla v(x)|^{2} d x\right\}^{\frac{1}{2}} \\
& \leq C\left\{\int_{B(0, C r)}|\nabla v(x)|^{2} d x\right\}^{\frac{1}{2}},
\end{aligned}
$$

and this completes the proof of (2.10).

Step 5. From (2.10) we see that $v$ admits a continuous extension $v(0)$ in $\mathbb{P}^{1}$ to the origin. We now prove that $\lim _{x \rightarrow 0} u_{11}(x)=0$ in coordinates with $v(0)= \pm(1,0)$, and hence that $u_{22}(x)=\triangle u(x)-u_{11}(x) \geq \frac{1}{2}$ in a neighbourhood of the origin. Indeed, if $x$ is fixed near the origin and if $\theta$ is the angle of rotation that diagonalizes $D^{2} u$ at $x$, i.e.

$$
R_{-\theta} D^{2} u(x) R_{\theta}=\left[\begin{array}{ll}
a & 0 \\
0 & d
\end{array}\right], \quad a \approx k(x), d \approx 1,
$$

then (2.10) yields the estimate

$$
|\theta| \approx|v(x)-v(0)| \leq C\left\{\int_{B(0, C|x|)}|\nabla v|^{2}\right\}^{\frac{1}{2}} .
$$


Thus $u_{11}(x)$ is given by

$$
\begin{aligned}
& (1,0) D^{2} u(x)\left(\begin{array}{c}
1 \\
0
\end{array}\right) \\
= & (1,0)\left[\begin{array}{cc}
\cos \theta & -\sin \theta \\
\sin \theta & \cos \theta
\end{array}\right]\left[\begin{array}{ll}
a & 0 \\
0 & d
\end{array}\right]\left[\begin{array}{cc}
\cos \theta & \sin \theta \\
-\sin \theta & \cos \theta
\end{array}\right]\left(\begin{array}{l}
1 \\
0
\end{array}\right) \\
= & a \cos ^{2} \theta+d \sin ^{2} \theta \\
\approx & k(x)+\theta^{2}=O\left(|x|^{2 m}+\int_{B(0, C|x|)}|\nabla v|^{2}\right) .
\end{aligned}
$$

This completes the proof of Lemma 1 .

Proof of Theorem 1. As we mentioned, Theorem 1 follows from Lemma1 1 and from Theorem 3 and Remark 21 (ii) in [4] (one easily checks that this remark holds for smooth $k$ as in (1.4) $)$.

Proof of Theorem 2. From elliptic regularity of the Monge-Ampère equation in $n=$ 2 , the isometric embedding $X$ obtained in 6, 8 is smooth away from the null set of $k$. By the finite type assumption (1.4), the null set of $k$ is discrete. For each $p \in M$ with $k(p)=0$, we may assume that $X(p)=0$, that the $x y$-plane is the tangent plane of $X(M)$ at 0 , and moreover that it can be represented locally as a graph $(x, y, u(x, y))$ over the $x y$-plane. Then $u$ satisfies equation (1.3) with

$$
k(x, y)=\kappa(s, t)\left\{1+u_{x}^{2}+u_{y}^{2}\right\}^{2},
$$

where $s, t$ are variables on $M$ and hence functions of $x, y$. Moreover, we note that $k$ continues to satisfy condition (1.4) in the $x, y$ variables.

The only catch is that here we must derive the Calabi estimate (2.1) used in the proof of Lemma 1 above, and this is now complicated by the fact that $s$ and $t$ are not smooth functions of $x$ and $y$ at the origin. When $m=1$, the estimates obtained in [9] suffice, but for $m>1$ we will adapt the proof of (2.1) in [12 to the case at hand. To accomplish this we pick conformal coordinates near $p$ so that $g=\lambda(s, t)\left(d s^{2}+d t^{2}\right)$. Since the embedding $X$ is an isometry, we have

$$
\lambda(s, t)\left(d s^{2}+d t^{2}\right)=g=d x^{2}+d y^{2}+d u^{2} .
$$

Now write $s, t, u$ as functions of $x, y$. Then we have

$$
\begin{aligned}
& \lambda(s, t)\left[\left(s_{x}^{2}+t_{x}^{2}\right) d x^{2}+2\left(s_{x} s_{y}+t_{x} t_{y}\right) d x d y\right]+\left(s_{y}^{2}+t_{y}^{2}\right) d y^{2} \\
& \quad=\left(1+u_{x}^{2}\right) d x^{2}+2 u_{x} u_{y} d x d y+\left(1+u_{y}^{2}\right) d y^{2} .
\end{aligned}
$$

For any $q$ in a neighborhood of $p$, we may rotate $s, t$ so that the Jacobian matrix $\left[\frac{\partial(s, t)}{\partial(x, y)}\right]$ is triangular with

$$
s_{y}=0 \text { and }\left|s_{x}\right|,\left|t_{y}\right| \geq c>0 \text { at } q .
$$

We now equate components in (2.15) and then differentiate twice in the $x, y$ variables. Since $s, t$ are smooth in $x, y$ away from the origin with $\|s\|_{C^{1,1}}$ and $\|t\|_{C^{1,1}}$ controlled by $\|u\|_{C^{1,1}}$, the same holds for $\lambda$. Furthermore, $\lambda \geq c>0$, and thus there are bounded functions $h_{j}(x, y)$, with supremum bounds depending only on 
$c^{-1}$ and $\|u\|_{C^{1,1}}$, such that

$$
\begin{aligned}
s_{x} s_{x y y}+t_{x} t_{x y y} & =h_{1}+\lambda^{-1} u_{x} u_{x y y} \\
\left(s_{y} s_{x y y}+t_{y} t_{x y y}\right)+\left(s_{x} s_{y y y}+t_{x} t_{y y y}\right) & =h_{2}+\lambda^{-1} u_{y} u_{y x y}+\lambda^{-1} u_{x} u_{y y y}, \\
s_{y} s_{y y y}+t_{y} t_{y y y} & =h_{3}+\lambda^{-1} u_{y} u_{y y y}, \\
s_{x} s_{x x x}+t_{x} t_{x x x} & =h_{4}+\lambda^{-1} u_{x} u_{x x x} \\
\left(s_{y} s_{x x x}+t_{y} t_{x x x}\right)+\left(s_{x} s_{y x x}+t_{x} t_{y x x}\right) & =h_{5}+\lambda^{-1} u_{x} u_{y x x}+\lambda^{-1} u_{y} u_{x x x}, \\
s_{y} s_{y x x}+t_{y} t_{y x x} & =h_{6}+\lambda^{-1} u_{y} u_{y x x}, \\
s_{x} s_{x x y}+t_{x} t_{x x y} & =h_{7}+\lambda^{-1} u_{x} u_{x x y}, \\
s_{y} s_{y y x}+t_{y} t_{y y x} & =h_{8}+\lambda^{-1} u_{y} u_{y y x} .
\end{aligned}
$$

Note that we omitted the equation obtained by applying $\partial_{x} \partial_{y}$ to the components of $d x d y$. After regrouping the equations in (2.17) we get

$$
\begin{aligned}
s_{x} s_{x y y}+t_{x} t_{x y y} & =h_{1}+\lambda^{-1} u_{x} u_{x y y}, \\
s_{x} s_{y y y}+t_{x} t_{y y y} & =h_{2}-h_{8}+\lambda^{-1} u_{x} u_{y y y}, \\
s_{y} s_{y y y}+t_{y} t_{y y y} & =h_{3}+\lambda^{-1} u_{y} u_{y y y}, \\
s_{x} s_{x x x}+t_{x} t_{x x x} & =h_{4}+\lambda^{-1} u_{x} u_{x x x}, \\
s_{y} s_{x x x}+t_{y} t_{x x x} & =h_{5}-h_{7}+\lambda^{-1} u_{y} u_{x x x}, \\
s_{y} s_{y x x}+t_{y} t_{y x x} & =h_{6}+\lambda^{-1} u_{y} u_{y x x}, \\
s_{x} s_{x x y}+t_{x} t_{x x y} & =h_{7}+\lambda^{-1} u_{x} u_{x x y}, \\
s_{y} s_{y y x}+t_{y} t_{y y x} & =h_{8}+\lambda^{-1} u_{y} u_{y y x} .
\end{aligned}
$$

From (2.18) and (2.16) we first obtain $t_{y} t_{i j k}=C^{i j k}+\lambda^{-1} u_{y} u_{i j k}$, where $t_{i j k}=$ $\frac{\partial^{3} t}{\partial x_{i} \partial x_{j} \partial x_{k}}, x_{1}=x, x_{2}=y$, for all $i, j, k=1,2$, and the $C^{i j k}$ are under control. Since $\left|t_{y}\right| \geq c>0$, we have $\left|t_{i j k}\right| \leq C\left(1+u_{i j k}\right)$. Plugging this back into (2.18) and using $\left|s_{x}\right| \geq c>0$, we obtain

$$
\left|s_{i j k}\right| \leq C\left(1+\left|u_{i j k}\right|\right), \quad\left|t_{i j k}\right| \leq C\left(1+\left|u_{i j k}\right|\right), \quad \text { for all } i, j, k=1,2,
$$

at the point $q$. With this, the proof of the Calabi estimate (2.1) for degenerate Monge-Ampére equations in [12] can now be adapted to the case at hand. Here is a sketch of the details in the case where $k=\kappa(s(x, y), t(x, y))$ is independent of $u$ and $D u$ in (1.3). The general case (2.13) is similarly adapted to the corresponding argument in [12, and is left to the reader.

Using the notation of [12, we obtain from the section on Calabi's identity there the inequality

$$
\frac{1}{2 n} \sigma^{2} \leq L \sigma+2 \sqrt{\sigma} \sqrt{\sum_{k p r} \frac{(\log \psi)_{k p r}^{2}}{u_{k k} u_{p p} u_{r r}}}+3 n \sigma \sup _{k \ell}\left|\frac{(\log \psi)_{k \ell}}{\sqrt{u_{k k} u_{\ell \ell}}}\right|,
$$

where $u$ satisfies (1.3), the second order linear operator $L$ is given by

$$
L w=u^{i j} w_{i j},
$$

the square $\sigma$ of the third order derivatives of $u$ is given by

$$
\sigma=u^{k \ell} u^{p q} u^{r s} u_{k p r} u_{\ell q s}
$$


and the function $\psi$ satisfies

$$
\psi(x, y)=k(s(x, y), t(x, y))
$$

with $s$ and $t$ as in (2.14). In coordinates that diagonalize $D^{2} u$ at a point, these definitions simplify to

$$
L w(x)=\frac{w_{i i}(x)}{u_{i i}(x)}, \quad \sigma(x)=\frac{u_{k p r}(x)^{2}}{u_{k k}(x) u_{p p}(x) u_{r r}(x)} .
$$

Now from [12, we also have the estimates

$$
\sqrt{\sum_{k p r} \frac{(\log \psi)_{k p r}^{2}}{u_{k k} u_{p p} u_{r r}}} \leq C \omega_{\psi}^{-3} \psi^{-\frac{3}{2}}, \sup _{k \ell}\left|\frac{(\log \psi)_{k \ell}}{\sqrt{u_{k k} u_{\ell \ell}}}\right| \leq C \widetilde{\omega}_{\psi}^{-2} \psi^{-1},
$$

where

$$
\begin{aligned}
& \omega_{\psi}(x, y)^{-1}=\left|\frac{D \psi(x, y)}{\psi(x, y)}\right|+\left|\frac{D^{2} \psi(x, y)}{\psi(x, y)}\right|^{\frac{1}{2}}+\left|\frac{D^{3} \psi(x, y)}{\psi(x, y)}\right|^{\frac{1}{3}}+|(x, y)|^{-1}, \\
& \widetilde{\omega}_{\psi}(x, y)^{-1}=\left|\frac{D \psi(x, y)}{\psi(x, y)}\right|+\left|\frac{D^{2} \psi(x, y)}{\psi(x, y)}\right|^{\frac{1}{2}}+|(x, y)|^{-1} .
\end{aligned}
$$

Since derivatives of $s$ and $t$ of order at most two are bounded, we obtain

$$
\sup _{k \ell}\left|\frac{(\log \psi)_{k \ell}}{\sqrt{u_{k k} u_{\ell \ell}}}\right| \leq C \widetilde{\omega}_{k}^{-2} k^{-1} \leq C \omega_{k}^{-2} k^{-1} .
$$

The only third derivatives of $s, t$ with respect to $x, y$ that appear in $\nabla_{x, y}^{3} \psi$ are the terms in $k_{s} \nabla_{x, y}^{3} s+k_{t} \nabla_{x, y}^{3} t$. Thus using (2.19) together with the chain rule yields

$$
\left|(\log \psi)_{k p r}\right| \leq C \omega_{k}^{-3}+C\left|\frac{D k}{k}\right|\left(1+\left|u_{k p r}\right|\right),
$$

and so

$$
\begin{aligned}
\sqrt{\sum_{k p r} \frac{(\log \psi)_{k p r}^{2}}{u_{k k} u_{p p} u_{r r}}} & \leq C \omega_{k}^{-3} k^{-\frac{3}{2}}+C \sqrt{\sum_{k p r} \frac{\left|\frac{D k}{k}\right|^{2}\left(1+\left|u_{k p r}\right|\right)^{2}}{u_{k k} u_{p p} u_{r r}}} \\
& \leq C \omega_{k}^{-3} k^{-\frac{3}{2}}+C \omega_{k}^{-1} \sqrt{\sum_{k p r} \frac{1}{u_{k k} u_{p p} u_{r r}}+\sigma} \\
& \leq C \omega_{k}^{-3} k^{-\frac{3}{2}}+C \omega_{k}^{-1}\left\{k^{-\frac{3}{2}}+\sqrt{\sigma}\right\} \\
& \leq C \omega_{k}^{-3} k^{-\frac{3}{2}}+C \omega_{k}^{-1} \sqrt{\sigma} .
\end{aligned}
$$

Plugging these estimates into (2.20) yields

$$
\begin{aligned}
\sigma^{2} & \leq 2 n L \sigma+C \sqrt{\sigma}\left(\omega_{k}^{-3} k^{-\frac{3}{2}}+\omega_{k}^{-1} \sqrt{\sigma}\right)+C \sigma \omega_{k}^{-2} k^{-1} \\
& \leq 2 n L \sigma+C \sqrt{\sigma} \omega_{k}^{-3} k^{-\frac{3}{2}}+C \sigma \omega_{k}^{-2} k^{-1},
\end{aligned}
$$

which is identical to the estimate obtained in [12]. 
Using Taylor's formula and (1.4) we can write for any smooth $k(X, r, \mathbf{p})$ with $X=(x, y)$

$$
\begin{aligned}
k(X, r, \mathbf{p}) & =\sum_{\ell=0}^{2 m} \frac{\left(X \cdot \nabla_{X}\right)^{\ell}}{\ell !} k(0, r, \mathbf{p})+\int_{0}^{1} \frac{(1-t)^{2 m}}{(2 m) !}\left(X \cdot \nabla_{X}\right)^{2 m+1} k(t X, r, \mathbf{p}) d t \\
& =\frac{\left(X \cdot \nabla_{X}\right)^{2 m}}{(2 m) !} k(0, r, \mathbf{p})+\int_{0}^{1} \frac{(1-t)^{2 m}}{(2 m) !}\left(X \cdot \nabla_{X}\right)^{2 m+1} k(t X, r, \mathbf{p}) d t,
\end{aligned}
$$

from which we obtain

$$
\left|\frac{\partial^{(|\alpha|+\beta+|\gamma|)}}{\partial x^{\alpha_{1}} \partial y^{\alpha_{2}} \partial r^{\beta} \partial \mathbf{p}^{\gamma}} k(x, y, r, \mathbf{p})\right| \leq C_{L}|(x, y)|^{2 m-|\alpha|},
$$

for $0 \leq|\alpha|+\beta+|\gamma| \leq 3$ in compact subsets $L$ of $\Omega \times \mathbb{R} \times \mathbb{R}^{2}$. This establishes inequality (13) in 12 for such $k$, and the remainder of the proof in [12] is unchanged. This completes our sketch of the proof of (2.1) in the case at hand.

Thus Lemma 1 holds in this setting, and together with the regularity result in [4], we obtain that $u$ is $C^{3, \alpha}$ in the $x, y$ variables for some $\alpha>0$. In turn then $X \in C^{3, \alpha}$, and $s, t$ are $C^{3, \alpha}$ in the $x, y$ variables, and finally $k$ is $C^{3, \alpha}$ in the $x, y$ variables. Higher regularity of $X$ now follows directly from [4].

\section{REFERENCES}

[1] Y.D. Burago and S.Z. Shefel, The geometry of surfaces in Euclidean spaces, Geometry, III, Encyclopaedia Math. Sci. 48, Springer, Berlin, 1992. MR.1306734

[2] L. Caffarelli, L. Nirenberg and J. Spruck, The Dirichlet problem for nonlinear second order elliptic equations, I. Monge-Ampère equations, Comm. Pure Appl. Math. 37 (1984), 369-402. MR739925 (87f:35096)

[3] P. Daskalopoulos And O. Savin, On Monge-Ampère equations with homogeneous right hand side, preprint, June 2007, arXiv:0706.3748.

[4] P. Guan, Regularity of a class of quasilinear degenerate elliptic equations, Advances in Mathematics 132 (1997), 24-45. MR 1488238 (99a:35068)

[5] P. Guan, $C^{2}$ a priori estimates for degenerate Monge-Ampère equations, Duke Math. J. 86 (1997), 323-346. MR1430436 (98d:35074)

[6] P. Guan And Y.Y LI, On Weyl problem with nonnegative Gauss curvature, Journal of Differential Geometry 39 (1994), 331-342. MR1267893 (95c:53051)

[7] P. Guan, N. S. Trudinger and X.-J. Wang, On the Dirichlet problem for degenerate Monge-Ampère equations, Acta Math. 182 (1999), 87-104. MR.1687172 (2000h:35051)

[8] J. Hong AND C. ZuiLY, Isometric embedding of the 2-sphere with nonnegative curvature in $\mathbb{R}^{3}$, Math. Z. 219 (1995), no. 3, 323-334. MR1339708 (96e:53005)

[9] J. IAIA, Isometric embeddings of surfaces with nonnegative curvature in $\mathbb{R}^{3}$, Duke Math. J. 67 (1992), 423-459. MR1177314 (93i:53004)

[10] L. Nirenberg, The Weyl and Minkowski problems in differential geometry in the large, Comm. Pure and Appl. Math. 6 (1953), 337-394. MR0058265 (15:347b)

[11] C. Rios, E. Sawyer and R. L. Wheeden, A higher-dimensional partial Legendre transform, and regularity of degenerate Monge-Ampère equations, Advances in Mathematics 193 (2005), 373-415. MR2137289 (2006f:35099)

[12] C. Rios, E. SAWyer And R. L. Wheeden, Regularity of subelliptic Monge-Ampère equations, to appear in Advances in Mathematics.

Department of Mathematics and Statistics, McGill University, Montreal, Quebec, CANADA H3A 2K6

E-mail address: guan@math.mcgill.ca

Department of Mathematics and Statistics, McMaster University, Hamilton, OnTARIO, CANAdA L8S $4 \mathrm{~K} 1$

E-mail address: sawyer@mcmaster.ca 